\title{
Escalas de avaliação de risco de quedas
}

\author{
Fall risk assessment tools \\ Escalas de evaluación del riesgo de caída \\ Maria José Martins da Costa-Dias* \\ Pedro Lopes Ferreira**
}

\section{Resumo}

Enquadramento: Os acidentes por quedas do doente continuam a ser os mais relatados dos incidentes a nível hospitalar. É um problema de qualidade e segurança do doente que a organização hospitalar deve ter em consideração. Os enfermeiros necessitam de avaliar o risco de queda e esta avaliação deve basear-se na utilização de escalas, mas os enfermeiros por vezes desconhecem qual o instrumento apropriado que devem utilizar.

Objetivo: Proporcionar informação sobre duas das escalas de avaliação do risco de quedas mais utilizadas, a St Thomas's Risk Assessment Tool in Falling Elderly Inpatients (STRATIFY) e a Morse Fall Scale (MFS).

Principais tópicos em análise: Efetuou-se uma análise dos instrumentos de avaliação do risco de queda, que foram alvo de maior número de revisões sistemáticas e que foram submetidas a validação prospetiva em dois ou mais coortes, com testes apropriados de predição da validade. Os resultados da pesquisa permitem concluir que ambas as escalas identificam as pessoas que têm risco de queda em função das suas características intrínsecas ou clínicas.

Conclusão: Os hospitais devem utilizar instrumentos já desenvolvidos e testados, como forma de comparar os seus dados, e estes devem ser submetidos a processos de adaptação cultural e linguística e de validação para a língua Portuguesa.

Palavras-chave: acidentes por quedas; serviços hospitalares; adulto; escalas.

\section{Abstract}

Background: Accidental falls remain the most reported incidents in hospital settings. This is a quality and safety issue for the patient which needs to be addressed by the hospital organisation. Nurses need to assess the risk of falling using scales. However, nurses are sometimes unsure of what is the most appropriate tool.

Objective: To provide information on the two most commonly used scales for assessing the risk of falling, i.e., the St Thomas's Risk Assessment Tool in Falling Elderly Inpatients (STRATIFY) and the Morse Fall Scale (MFS).

Main topics for analysis: The fall risk assessment tools which were the subject of more systematic reviews and prospective validation in two or more cohorts, with appropriate tests to predict their validity, were analysed. The review concluded that both scales identify patients who are at risk of falling based on their intrinsic or clinical characteristics.

Conclusion: Hospitals should use scales which have already been developed and tested for data comparison. These scales should be culturally and linguistically adapted, and validated for the Portuguese language.

Keywords: accidental falls; hospital services; adult; scales.

* Enfermeira Especialista em Saúde Infantil e Pediátrica. Aluna do Doutoramento em Enfermagem do Instituto de Ciências da Saúde da Universidade Católica Portuguesa Hospital da Luz, 100. 1500-650, Lisboa, Portugal. [cdias@hospitaldaluz.pt]. Morada para correspondência: Rua Basílio Teles, N. ${ }^{\circ} 11,2 .{ }^{\circ}$ Esq., 2720-063, Amadora, Portugal. ** Ph.D.University of Wisconsin-Madison, EUA. Professor Associado com Agregação. Director do Centro de Estudos e Investigação em Saúde da Universidade de Coimbra. Faculdade de Economia. Centro de Estudos e de Investigação em Saúde. Universidade de Coimbra, 165. 3004-512, Coimbra, Portugal [pedrof@fe.uc.pt].

\section{Resumen}

Marco contextual: Los accidentes por caídas de pacientes siguen siendo los más comunicados a nível hospitalario. Es una cuestión de calidad y seguridad del paciente que la organización del hospital tiene que abordar. Los enfermeros han de evaluar el riesgo de caída, y esta evaluación debe basarse en el uso de escalas. No obstante, los enfermeros a veces no saben qué escala es la adecuada. Objetivos: Proporcionar información sobre dos de las escalas para evaluar el riesgo de caídas que más se utilizan, la $S t$ Thomas's Risk Assessment Tool in Falling Elderly Inpatients (STRATIFY) y la Escala de Caídas de Morse (MFS).

Principales temas de análisis: Se llevó a cabo un revisión de las escalas de evaluación del riesgo de caídas que han tenido un mayor número de revisiones sistemáticas y que han sido sometidas a una validación prospectiva en dos o más cohortes, con pruebas adecuadas de predicción de la validez. Los resultados de la investigación, permiten concluir que ambas escalas identifican a los pacientes que están en riesgo de caída en función de sus características intrínsecas o clínicas.

Conclusión: Los hospitales deben utilizar escalas ya desarrolladas y probadas, como forma de comparar sus datos. Asimismo, estas escalas deben ser sometidas a procesos de adaptación cultural y lingüística y de validación al portugués.

Palabras clave: accidentes por caídas; servicios hospitalarios; adulto; escalas.

Recebido para publicação em: 14.10.12

Aceite para publicação em: 28.02 .14 


\section{Introdução}

O acidente mais notificado a nível hospitalar e nos cuidados continuados é a queda do doente, da qual cerca de 5\% resulta em fraturas e 5\% a 11\% em outros danos graves (Perell, Nelson, Goldman, Preito-Lewis \& Rubenstein, 2001). Apesar dos avanços no processo de compreensão das quedas, estas continuam a ser um problema importante (Almeida, Abreu, \& Mendes, 2010). Nos Estados Unidos, a nível da comunidade, as quedas foram identificadas como a segunda causa de morte acidental e $75 \%$ das quedas ocorrem em idosos (Morse, 2009). Em Portugal, a nível hospitalar, existem estudos que apontam para uma prevalência na pessoa adulta internada de 1,5\% (Pina et al., 2010).

Entre janeiro de 2006 e janeiro de 2008 (25 meses) foram analisadas 4200 quedas associadas a acidentes a nível hospitalar, com macas, camas, cadeiras e problemas no piso e nas casas de banho pela Inspeção Geral das Atividades em Saúde Portuguesa (Soares \& Almeida, 2008).

Foram questionados 67 hospitais do Serviço Nacional de Saúde, mas apenas 56 notificaram quedas nos doentes, o que significa que 11 não sinalizaram quaisquer acidentes, ou não existem registos. Nesta análise, as quedas que resultaram em óbitos constituíram apenas 2\% do total de quedas. Em 29 casos $(0,7 \%)$, foram originados processos de natureza disciplinar. Os dados permitiram concluir que apenas 2\% dos acidentes ocorreram na urgência, a que se juntaram outros $2 \%$ em local indeterminado. A maioria das situações (4022) ocorreu nas restantes áreas, sobretudo no internamento e a maioria das quedas sinalizadas foram de camas ou macas mas incluiu, no total de acidentes, as quedas nas casas de banho, cadeirões, cadeiras de rodas e em pisos escorregadios (Soares \& Almeida, 2008).

O tempo de internamento hospitalar é muitas vezes prolongado devido às consequências causadas por uma queda (Cumming, Sherrington, \& Lord, 2008; Oliver, Britton, Seed, Martin, \& Hopper, 1997), o que induz, necessariamente, custos adicionais ao sistema de saúde, e naturalmente, mais desconforto e sofrimento aos doentes.

As quedas estão também associadas a níveis de ansiedade e de depressão mais elevados, perda de confiança e ao síndroma pós-queda (Oliver, Daly, Martin, \& McMurdo, 2004; Perell et al., 2001). As quedas não são, em princípio, só onerosas para 0 doente e para as organizações de saúde, mas resultam também em sentimentos de ansiedade e culpa entre os profissionais que nelas trabalham, formalização de reclamações e em litígio com os doentes e as famílias (Healey \& Scobie, 2007; Oliver et al., 2004).

A avaliação do risco de queda é um dos indicadores de avaliação da qualidade hospitalar, no que se refere à segurança do doente, sobretudo aos doentes com 65 ou mais anos. Torna-se necessário que as organizações de saúde identifiquem quais os instrumentos disponíveis, e devidamente estudados, que permitam uma correta avaliação do risco de queda, como primeira etapa de desenvolvimento de um programa de prevenção de quedas em contexto hospitalar, sendo esta a questão central deste artigo.

\section{Dissertação}

Quando hospitalizadas, as pessoas idosas estão duplamente vulneráveis, por causa da idade, enfraquecidos pelo processo de doença ou por uma cirurgia, por permanecerem mais tempo na cama e estarem mais medicados. São colocados em ambientes estranhos e dependem da ajuda de outros para efetuarem as suas atividades diárias.

Contudo, podemos considerar que não é só a prevalência de quedas nas pessoas hospitalizadas que é importante, mas também o grau de dano que se encontra associado (Morse, 2009). Por causa destes danos, quer nas pessoas quer na sociedade, diversas pesquisas tem vindo a ser desenvolvidas, no sentido de se criarem programas de prevenção de quedas, cujo passo inicial é a avaliação do risco de queda (Morse, 2009).

A nível dos vários locais de trabalho e, em particular a nível hospitalar, os gestores de cuidados de enfermagem interrogam-se frequentemente sobre qual o instrumento de avaliação a utilizar para avaliar o risco de queda. Isto porque os instrumentos de medida devem ter qualidades essenciais como a fiabilidade e a validade. Além disto, todas as escalas que existem estão concebidas em outra língua que não a portuguesa e para outra cultura e, por isso, têm de ser submetidas a um processo de tradução, adaptação cultural e linguística e validação para a língua portuguesa, para que os instrumentos de medida se mantenham fiéis ao original (Wild et al., 2005). Por fim, quando se escolhe um método de avaliação das 
quedas dos doentes, é importante que o instrumento seja aplicado tal como foi concebido e publicado pelo autor que o desenvolveu, pois alterações dos itens, ou de formas de aplicação, podem destruir a fiabilidade e a validade de uma escala (Morse, 2009).

As escalas de avaliação de risco de quedas são ferramentas que atribuem valores numéricos a diversos factores de risco (Healey \& Scobie, 2007). O somatório destes fatores predizem se o doente tem um risco de queda baixo, médio ou elevado (Morse, 2009). Existem diversos estudos descritivos de avaliação do risco de queda, mas apenas cinco instrumentos, foram testados quanto à forma como predizem as quedas (Healey \& Scobie, 2007). Esses instrumentos são o Score de Innes (1985), a Escala de Quedas de Morse (Morse, Morse \& Tylko, 1989), o Score de Schmid (1990), o índice de Downton (Nyberg \& Gustafson, 1996) e a avaliação do risco STRATIFY (Oliver et al.,1997), mas apenas dois foram testadas em diferentes grupos de doentes, fora do estudo de investigação original (Oliver et al., 2004), a Escala de Quedas de Morse e a avaliação do risco STRATIFY (Tabela 1).

Tabela 1

Escalas de avaliação de quedas

\begin{tabular}{lll}
\hline Nome do instrumento & Autores & Ano \\
\hline Innes Score & Else M. Innes & 1985 \\
Morse Fall Scale & Janice M. Morse; Robert M. Morse; Suzanne J. Tylko & 1989 \\
Schmid Score & Nancy A. Schmid & 1990 \\
Downton Index & L. Nyberg; Y. Gustafson & 1996 \\
Stratify & D. Oliver; M. Britton; P. Seed; F.C. Martin; A.H. Hopper & 1997 \\
\hline
\end{tabular}

A literatura alerta para o facto de, mesmo instrumentos validados poderem falhar na previsão de um número significativo de quedas (Oliver et al., 2004). Isto acontece devido a nem sempre os fatores que contribuem para as quedas fazerem parte dos indicadores desses instrumentos. No entanto, existe evidência de que uma pessoa que já tenha caído, tem mais risco de voltar a cair nas mesmas circunstâncias, o mesmo acontecendo em doentes que apenas caíram uma vez durante o internamento, sendo importante intervir a nível dos factores de risco que são preveníveis ou reversíveis (Morse, 2009).

\section{Metodologia}

Efetuou-se uma análise dos dois instrumentos de avaliação do risco de queda que foram alvo de maior número de revisões sistemáticas e que foram submetidos a validação prospetiva em dois ou mais coortes, com testes apropriados de predição da validade (Morse, Tylko, \& Dixon,1987; Healey \& Scobie, 2007). Investigou-se também se foram submetidas às etapas do processo de adaptação cultural e linguística e de validação para a língua Portuguesa. O objetivo desta pesquisa foi, então, proporcionar informação sobre as duas escalas de avaliação do risco de quedas mais utilizadas, a St Thomas's Risk Assessment Tool in Falling Elderly Inpatients (STRATIFY) e a Escala de Quedas de Morse (MFS).

Apresentam-se, de seguida, as características principais destas duas escalas.

\section{Escala STRATIFY}

Foi concebida em 1997 por David Oliver e seus colaboradores em Inglaterra (Oliver et al., 1997) e desenvolveu-se em três fases. Na primeira fase da sua conceção identificaram-se as características clínicas dos doentes internados (com 65 ou mais anos) que predizem a possibilidade de ocorrer uma queda. $\mathrm{O}$ estudo foi conduzido com o objetivo de identificar os fatores de risco que podiam ser claramente avaliados pelos enfermeiros como parte de um instrumento de avaliação de rotina. Nesta fase identificaram-se quais os fatores de risco que tinham uma associação significativa com a ocorrência das quedas. Numa segunda e terceira fases usaram-se as características identificadas nos doentes para se elaborar uma ferramenta de avaliação do risco e avaliar a sua potencialidade para predizer as quedas dos doentes. Para a primeira fase foi desenhado um estudo prospetivo do tipo casos-controlos, com 116 casos 
e 116 controlos e, para as segunda e terceira fases, foi desenhado um outro estudo com 271 doentes de avaliação prospetivado para analisar a forma como a escala foi capaz de predizer o risco de queda em dois coortes. As duas primeiras fases decorreram em unidades de idosos do St Thomas's Hospital em Londres, um hospital universitário de 700 camas, das quais 96 são dedicadas à área geriátrica, distribuídas por quatro unidades, sendo uma destinada à reabilitação dos doentes com acidentes vasculares cerebrais. A terceira fase decorreu no Kent and Canterbury Hospital, um hospital geral de 500 camas no qual se incluem duas unidades de internamento em situação aguda e quatro de cuidados continuados para idosos e onde foram estudados 331 doentes.

A nível das características clínicas dos doentes foram avaliadas 21 características na primeira fase, incluindo um teste mental abreviado, o índice de Barthel modificado, uma avaliação da atividade de transferência e de mobilidade, obtidas através da conjugação das secções do auto cuidado no índice de Barthel e diversas avaliações realizadas por enfermeiros. Através da regressão logística, foram identificados cinco fatores associados independentemente a um elevado risco de queda, com base no cálculo de odds ratios (OR): diagnóstico de queda $(O R=4,64 ; I C 95 \%=2,59-8,33)$, pontuação de transferência e mobilidade entre três e quatro $(O R=2,10 ; \quad$ IC95\% =1,22-3,61), avaliação do enfermeiro se o doente está agitado (OR=20,9;

\section{Tabela 2}

\section{Perguntas da escala de avaliação de risco STRATIFY}

IC95\% $=9,62-45,62)$, necessidade de utilizar as instalações sanitárias com frequência $(\mathrm{OR}=2,48$; $I C=95 \%=1,08-5,70)$ ou se tem dificuldades de visão $(O R=3,56 ;$ IC $95 \%=1,26-10,05)$.

Foi definida uma avaliação de risco atribuindo-se a cada um destes fatores uma pontuação de um dentro de uma possibilidade de um a cinco. Na fase dois e três foi testado o ponto de corte de definição de risco elevado, tendo sido aferido o valor igual ou superior a dois, como o ponto de corte adequado.

Os autores (Oliver et al., 1997) concluíram também que esta escala de avaliação de risco é simples e prediz com sensibilidade e especificidade clínica uma elevada percentagem de quedas nas pessoas idosas internadas. A sensibilidade e especificidade foram calculadas em mais de $80 \%$ nos dois coortes de doentes.

A escala STRATIFY é constituída por cinco perguntas com opções dicotómicas (sim/não) de resposta (Tabela 2). No entanto, na última pergunta, a pontuação é obtida através da conjugação de duas respostas do índice de Barthel modificado em relação, respetivamente, à capacidade do doente em se transferir da cama para uma cadeira (0: Incapaz; 1: Necessita de ajuda significativa; 2: Necessita de ajuda mínima; 3: Independente) e ao nível de mobilidade do doente (0: Imóvel; 1 : Independente com a ajuda de uma cadeira de rodas; 2: Usa apoios de marcha ou caminha com a ajuda de uma pessoa; 3 : Independente).

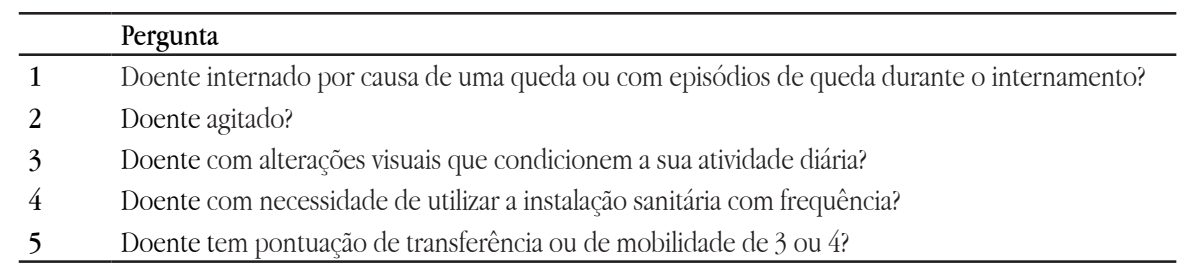

A pontuação total da escala STRATIFY é obtida com base na soma das respostas referentes às cinco perguntas, podendo tomar valores entre zero e cinco. Uma pontuação igual a 0 corresponde a um risco considerado baixo, igual a 1 corresponde a um risco moderado e, por fim, superior ou igual a 2 corresponde a um risco elevado. Em resumo, podemos dizer que a STRATIFY é uma ferramenta de previsão de quedas, que foi desenvolvida para ser utilizada no hospital e em doentes adultos idosos, baseada em cinco itens, em que cada item tem uma pontuação de 1 (se presente) ou 0 (se ausente), com um ponto de corte igual ou superior a 2 no total da escala.

Tem sido muito utilizada como parte de um programa de prevenção de quedas, mas a sua utilidade não está muito clara em diversos cenários (Oliver et al., 2008). De facto, em 2008, cerca de 
10 anos depois da sua publicação e de um número significativo de estudos com vários coortes de doentes, foi realizada uma meta-análise dos artigos que envolviam a escala STRATIFY. Nesta investigação, foram identificados 41 artigos, dos quais oito foram eleitos para inclusão em revisão sistemática e quatro para meta análise. Concluíram os autores (Oliver et al., 2008) que a escala STRATIFY tem sido aplicada em diversos estudos de validação e é comparável a outras escalas em tempo de aplicação, adesão e fiabilidade. Embora com valores elevados para a especificidade e valor preditivo negativo, a sensibilidade e o valor preditivo positivo foram geralmente baixos, de forma a fazer deste instrumento um indicador útil na identificação dos doentes de alto risco para prevenção de quedas a nível hospitalar (Tabela 3).

A meta-análise referida demonstra também que o tipo da população e o tipo de unidades em que é aplicada podem afetar o desempenho da escala STRATIFY.

A escala foi testada com modificações do peso atribuído aos diferentes itens, em dois hospitais em Hamilton, Ontário no Canadá, passando a pontuação total original da escala de 5 pontos para 30 pontos. Neste estudo foi obtido um coeficiente de correlação intraclasse de 0,78 , uma sensibilidade de $91 \%$ e especificidade de $60 \%$, para o ponto de corte 9, no entanto a alteração da pontuação da escala não foi validada em outros estudos (Papaioannou et al., 2004).

Tabela 3

Resumo da validação da escala STRATIFY

\begin{tabular}{|c|c|c|c|c|}
\hline Estudo & Sensibilidade ${ }^{1}$ & Especificidade ${ }^{2}$ & $\begin{array}{c}\text { Valor preditivo } \\
\text { positivo }^{3}\end{array}$ & $\begin{array}{c}\text { Valor preditivo } \\
\text { negativo }^{4}\end{array}$ \\
\hline $\begin{array}{l}\text { Oliver et al. (1997) } \\
\text { St Thomas's Hospital ( Fase 2) }\end{array}$ & $93,0 \%$ & $87,7 \%$ & $62,3 \%$ & $98,3 \%$ \\
\hline $\begin{array}{l}\text { Oliver et al. (1997) } \\
\text { St Thomas's sospital (Fase 3) }\end{array}$ & $54,4 \%$ & $87,6 \%$ & $48,9 \%$ & $89,8 \%$ \\
\hline $\begin{array}{l}\text { Oliver (2008) } \\
\text { Meta análise de } 4 \text { estudos }\end{array}$ & $67,2 \%$ & $51,2 \%$ & $23,1 \%$ & $86,5 \%$ \\
\hline Papaiannou et al. (2004) & $91,2 \%$ & $60,2 \%$ & - & - \\
\hline
\end{tabular}

${ }^{1}$ Sensibilidade $=$ Probabilidade de um doente identificado de alto risco cair entre todos os doentes que caíram.

${ }^{2}$ Especificidade $=$ Probabilidade de um doente identificado de baixo risco não cair entre todos os doentes que não caíram.

${ }^{3}$ Valor preditivo positivo $=$ Probabilidade de um doente identificado de alto risco cair entre todos os doentes identificados com alto risco.

${ }^{4}$ Valor preditivo negativo $=$ Probabilidade de um doente identificado de baixo risco não cair entre todos os doentes identificados com baixo risco.

${ }^{5}$ Odds ratio $=$ Probabilidade de queda em doentes com risco elevado versus probabilidade de queda em doentes com baixo risco.

\section{Escala de Quedas de Morse}

Foi desenvolvida em 1985 no Canadá, por Janice M. Morse, com base num estudo prospetivo, com o objetivo de identificar e predizer as pessoas com risco de quedas fisiológicas, em 100 doentes que caíram e em 100 doentes que não caíram, como grupo de controlo. O estudo foi realizado num hospital de doentes agudos com 1200 camas, com um centro geriátrico com 50 camas e um centro para veteranos com 140 camas, tendo sido excluídos todos os doentes das unidades pediátricas e com menos de 18 anos (Morse et al., 1987). Trata-se de uma escala que foi concebida para ser aplicada através de entrevistas com os doentes e da consulta dos processos clínicos, com o objetivo de avaliar o risco de queda. Tem um tempo estimado de preenchimento de menos de três minutos.
Através da Tabela 4 pode verificar-se que a MFS (Morse et al., 1989) apresentou uma sensibilidade de $72,0 \%$, uma especificidade de $83,0 \%$, um valor preditivo positivo de $10,3 \%$ e um valor preditivo negativo de $99,2 \%$. A fiabilidade da escala foi avaliada como excelente, através do coeficiente de correlação intraclasse (CCI $\geq 0,75)$ com base na avaliação de seis doentes por 21 enfermeiros, sendo o seu valor de 0,96 , indicando uma boa reprodutibilidade. Os coeficientes individuais para cada uma das perguntas da escala foram respetivamente de 1,0 para as perguntas um e quatro, de 0,99 para a pergunta dois, de 0,98 para a pergunta três e de 0,82 para a pergunta 5, não tendo sido avaliado o coeficiente para a pergunta 6.

A escala foi avaliada por Schwendimann, De Geest e Milisen (2006), em duas unidades de medicina 
interna de um hospital suiço, sendo o coeficiente de correlação intraclasse de 0,68 . A sensibilidade variou entre $91,5 \%$ e $38,3 \%$, a especificidade entre $81,7 \%$ e

Tabela 4

Resumo da validação da Escala de Morse
$10,6 \%$, o valor preditivo positivo entre $12,5 \%$ e $22,5 \%$ e o valor preditivo negativo entre $90,2 \%$ e $95,7 \%$.

\begin{tabular}{lccccc}
\hline Estudo & Sensibilidade & Especificidade & $\begin{array}{c}\text { Valor preditivo } \\
\text { positivo }\end{array}$ & $\begin{array}{c}\text { Valor preditivo } \\
\text { negativo }\end{array}$ & CCI $^{1}$ \\
\hline Morse, Morse e Tylko (1889) & $72,0 \%$ & $83,0 \%$ & $10,3 \%$ & $99,2 \%$ & 0,96 \\
Schwendimann, De Geest e & $74,5 \%$ & $65,8 \%$ & $23,2 \%$ & $94,9 \%$ & 0,68 \\
Milisen (2006) & & & &
\end{tabular}

${ }^{1}$ Coeficiente de correlação intraclasse

${ }^{2}$ Valores para o ponto de corte 55

A escala é composta por seis itens que refletem os fatores de risco de queda e que estão resumidos na Tabela 5. Estes seis itens foram identificados através do método estatístico de análise descriminante. A presença de fator de risco é assinalada como sim ou não para os itens 1, 2 e 4; para os outros três itens, a pontuação é baseada em dois ou três descritores. É atribuída uma pontuação de $0 \mathrm{a} 30$ pontos às respostas identificadas como sim ou não e aos descritores para cada item. A pontuação total da MFS varia entre 0 e 125 descriminando as pessoas em função do nível

Tabela 5

Perguntas da escala de avaliação de risco de Morse de risco de queda. Assim, considera-se que a pessoa não tem qualquer risco de queda se a pontuação total da MFS for inferior ou igual a 24, o que apenas exige intervenções básicas de enfermagem. Se a pontuação total estiver entre 25 e 50, considera-se que a pessoa tem um risco baixo de queda e são necessárias intervenções padrão de prevenção de quedas. Por fim, considera-se que a pessoa tem um risco elevado, se obtiver uma pontuação total superior ou igual a 51 e, neste caso, há que iniciar intervenções de prevenção de quedas de alto risco (Morse, 2009).

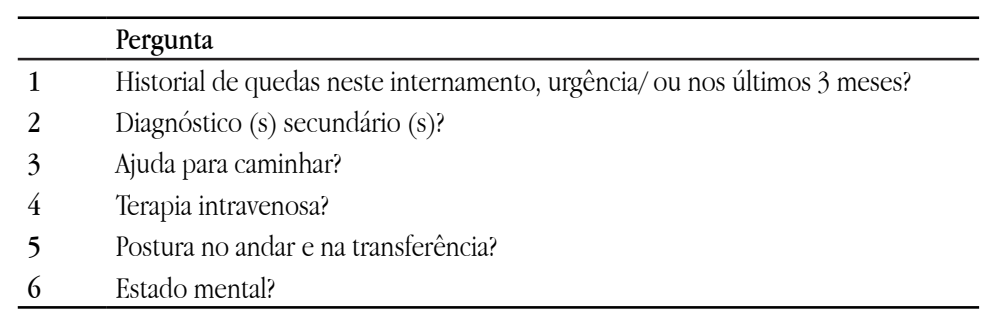

Morse (2009) alerta que a escala deve ser calibrada para cada realidade hospitalar ou unidade para que as estratégias de prevenção sejam dirigidas para os doentes com maior risco. Ou seja, os pontos de corte podem ser diferentes dependendo da utilização da escala num serviço de internamento de doentes agudos, de cuidados continuados ou de cuidados paliativos, dentro da mesma organização a escala pode ter pontos de corte diferentes.

Os instrumentos de avaliação do risco podem ter um papel fundamental como primeiro passo na implementação de um programa efetivo e eficiente de prevenção de quedas (Perell et al., 2001). Embora sendo vitais para a eficiência de um programa, estes têm sido criticados pela sua capacidade de, com precisão, identificarem o doente que efetivamente cai (Oliver, 2008).

As escalas STRATIFY e MFS identificam os doentes que têm risco de queda em função das suas características intrínsecas ou clínicas (estado mental, alterações da mobilidade, história anterior de quedas, frequência da eliminação/dependência, doença aguda ou crónica).

$\mathrm{Na}$ Tabela 6 apresenta-se um resumo comparativo entre ambas as escalas estudadas relativamente ao número de perguntas que compõem cada escala, população estudada, assim como anos em que 
foram criadas e em que países, dimensões comuns que integram, natureza dos estudos utilizados na construção dos dois instrumentos e método estatístico, países para os quais foi feita a adaptação cultural e linguística.

Como se pode observar na Tabela 6 , as escalas foram construídas em países diferentes, têm 12 anos de diferença sendo a MFS anterior à STRATIFY. A primeira foi construída por uma enfermeira e a segunda por um médico, revelando a preocupação, já existente na década dos anos 80 e 90, com as questões da segurança do doente associadas às quedas em contexto hospitalar.

Tabela 6

Resumo comparativo de ambas as escalas

\begin{tabular}{|c|c|c|}
\hline Características & Escala STRATIFY & Escala de Morse \\
\hline Número de perguntas & 5 & 6 \\
\hline Dimensões comuns & \multicolumn{2}{|c|}{$\begin{array}{c}\text { História anterior de quedas } \\
\text { Alteração do estado mental } \\
\text { Avaliação da capacidade de transferência, da mobilidade } \\
\text { e da utilização de apoios de marcha }\end{array}$} \\
\hline $\begin{array}{c}\text { Dimensões individuais de cada } \\
\text { escala }\end{array}$ & $\begin{array}{l}\text { Alterações visuais } \\
\text { Utilização frequente da instalação } \\
\text { Sanitária }\end{array}$ & $\begin{array}{l}\text { Existência de diagnóstico secundário } \\
\text { Presença de perfusões contínuas } \\
\text { intravenosas }\end{array}$ \\
\hline População estudada & Adultos com 65 e mais anos & Adultos com 18 ou mais anos \\
\hline Desenvolvimento & Três hospitais & Três hospitais \\
\hline Tipo de estudos utilizados & Casos-controlos e coorte & Casos-controlos \\
\hline Método estatístico de construção & Regressão logística & Análise descriminante \\
\hline $\begin{array}{l}\text { País e ano em foi validada e cons- } \\
\text { truída }\end{array}$ & Inglaterra, 1997 & Canadá, 1985 \\
\hline $\begin{array}{c}\text { Países com adaptação cultural e } \\
\text { linguística }\end{array}$ & $\begin{array}{c}\text { Austrália, Bélgica, Canadá, França, } \\
\text { Holanda, Itália. }\end{array}$ & $\begin{array}{l}\text { Alemanha, Correia, China, Dinamarca, } \\
\text { Espanha, Filipinas, França, Suíça, Japão, } \\
\text { Portugal }\end{array}$ \\
\hline
\end{tabular}

A sua dimensão é comparável (5 a 6 itens), sendo que a primeira apresenta um formato dicotómico de respostas e a segunda combina este formato com itens de escolha múltipla. As características avaliadas dos doentes são similares nas duas escalas e os fatores de risco intrínsecos comuns são o estado mental, a mobilidade e a história anterior de quedas.

Ambas as escalas foram desenvolvidas em ambiente hospitalar, são de fácil utilização, consomem relativamente pouco tempo no seu preenchimento e foram construídas para serem utilizadas no doente adulto. A MFS é mais abrangente do que a STRATIFY, pois destina-se ao adulto em geral enquanto a segunda está mais adaptada ao doente com 65 ou mais anos. O tipo de estudos utilizados na construção de ambas foram de natureza epidemiológica e a técnica estatística de previsão e explicação utilizada para selecionar as variáveis incluídas nas escalas, foi a análise descriminante para a construção da MFS e a regressão logística para a construção da STRATIFY. Ambas as escalas foram submetidas a um processos de adaptação cultural e linguística, mas apenas a MFS se encontra validada e adaptada cultural e linguisticamente para a língua portuguesa, sendo a escala mais divulgada e utilizada a nível nacional (Ordem dos Enfermeiros, 2010; Soares \& Almeida, 2008), tendo sido testada em diversos cenários para além daqueles em que foi desenvolvida e em diversas populações (Schwendimann et al., 2006), sendo o número de estudos efetuados e países onde se encontra feita a adaptação cultural e linguística superior quando comparada com a escala STRATIFY.

A escala STRATIFY sofreu modificações (Papaioannou et al., 2004) no peso atribuído aos seus itens, existindo assim uma variante da escala original, que não foi testada em outros estudos independentes, o que não aconteceu com a MFS.

Uma revisão sistemática com meta-análise, efetuada recentemente sobre os estudos que incidem na escala STRATIFY, conclui que a sua fiabilidade é limitada e que não deve ser utilizada isoladamente na avaliação de pessoas com elevado risco de queda, mas como parte de uma avaliação multifatorial. No entanto, numa pessoa com elevado risco de queda o critério 
de avaliação multifatorial deve ser sempre utilizado independentemente da escala que seja utilizada pela organização.

No que se refere à reprodutibilidade das escalas, apenas temos informação relativamente ao cálculo do coeficiente intraclasse da MFS, o qual revelou ser excelente.

No seu estudo original da STRATIFY, a unidade de análise foi o número de episódios de queda que ocorreram durante o estudo. Cada queda foi encarada como um novo episódio e as pessoas que caíram mais de uma vez foram incluídas diversas vezes, não sendo este desenho do estudo o ideal pois pode estar sujeito a introdução de viés na seleção dos controlos apropriados. No caso da MFS foram selecionadas pessoas com queda e sem queda.

As recomendações para a escolha apropriada de uma escala são uma elevada sensibilidade e especificidade, uma boa concordância entre observadores, uma implementação em populações similares àquelas em que os estudos se basearam ou foram realizados, existência de procedimentos escritos que orientem no uso da escala, um tempo razoável de preenchimento e critérios e limiares estabelecidos para o início de intervenções (Perell et al., 2001).

Estes instrumentos são utilizados pelos enfermeiros na admissão do doente e periodicamente atualizados (por turno, por dia, ou semanalmente), dependendo da situação do doente e das políticas e procedimentos definidas por cada organização. Por isso, nos hospitais, o tempo de preenchimento de uma escala é um critério crítico. Para além disto, a gravidade da situação do doente, as prescrições terapêuticas que afetam a mobilidade e o estado mental e cognitivo, exigem uma avaliação fácil e rápida sem ónus para o doente.

\section{Conclusão}

A avaliação do risco de queda ou a avaliação do doente em risco é um componente essencial de qualquer programa de prevenção de quedas. A sua finalidade é identificar quais os doentes em risco, com o objetivo de corrigir a situação e, finalmente, evitar a ocorrência de quedas.

Idealmente, a avaliação do risco de queda deve ser efetuada na admissão do doente e pelo menos de 3 em 3 dias durante o período de internamento, ou ainda quando é transferido para uma unidade diferente, quando ocorre uma mudança na sua condição, ou depois de uma queda.

Existem diversas ferramentas de avaliação disponíveis para a avaliação do risco de queda. Em ambiente de cuidados agudos, estas foram resumidos por Perell e colaboradores. Apesar de existirem diversas escalas, existe, concomitantemente, pouca informação sobre como escolher um instrumento apropriada à população em questão.

Recomenda-se que somente instrumentos em que foram testadas empiricamente a fiabilidade e a validade devam ser utilizados, os quais não substituem, mas complementam a avaliação do enfermeiro sobre o risco de queda.

Constata-se ainda que a MFS é a mais estudada a nível internacional e aplicada em diversos cenários e a nível nacional é a mais divulgada e utilizada, está especialmente concebida para o adulto em geral, podendo assim ser aplicada na larga maioria das organizações hospitalares portuguesas, necessitando no entanto de ser submetida a um processo de adaptação cultural e linguística e ainda validada para a nossa língua, processo que se espera que esteja concluído em breve.

Não existe, assim, necessidade de se desenvolverem escalas próprias, que podem mesmo ser contraproducentes para o objetivo geral de avaliação do risco, pois as escalas e as pontuações devem poder ser comparáveis entre as diversas organizações, de modo a garantir a validação de boas práticas.

\section{Referências bibliográficas}

Almeida, R. A., Abreu, C. C., \& Mendes, A. M. (2010). Quedas em doentes hospitalizados: Contributos para uma prática baseada na prevenção. Revista de Enfermagem Referência, 3(2), 163-172. doi:10.12707/RIII1016

Cumming, R., Sherrington, C., \& Lord, R. (2008). Cluster randomized trial of a targeted multifactorial intervention to prevent falls among older people in hospital. BMJ, 336(7647), 758-760. doi:10.1136/bmj.39499.546030.BE

Healey, F., \& Scobie, S. (2007). Slips, trips and falls in hospital: The third report from Patient Safety Observatory. London, England: National Patient Safety Agency.

Innes, E. M. (1985). Maintaining fall prevention. Quality Review Bulletin, 11(7), 217-221.

Morse, J. (2009). Preventing patients falls: Establishing a fall intervention program (2nd ed.). New York, NY: Springer. 
Morse, J. M., Morse, R. M., \& Tylko, S. J. (1989). Development of a scale to identify the fall-prone patient. Canadian Journal on Aging, 8(4), 366-377.

Morse, J. M., Tylko, S. J., \& Dixon, H. A. (1987). Characteristics of the fall-prone patient. The Gerontologist, 27(4), 516-522.

Nyberg, L., \& Gustafson, Y. (1996). Using the Downton index to predict those prone to falls in stroke rehabilitation. Stroke, 27(10), 1821-1824.

Oliver, D. (2008). Falls risk-prediction tools for hospital inpatients. Time to put them to bed? Age and Ageing, 37(3), 248-250. doi:10.1093/ageing/afn088

Oliver, D., Britton, M., Seed, P., Martin, F. C., \& Hopper, A. H. (1997). Development and evaluation of evidence based risk assement tool (STRATIFY) to predict which elderly inpatients will fall: Case-control and cohort studies. BMJ, 315(7115), 1049-1053.

Oliver, D., Daly, F., Martin, F., \& McMurdo, M. (2004). Risk factors and risk assessment tools for falls in hospital in-patients: A systematic review. Age and Ageing, 33(2), 122-130. doi:10.1093/ageing/afh017

Oliver, D., Papaioannou, A., Giangregorio, L., Thabane, L., Reizgys, K., \& Foster, G. (2008). A systematic review and meta-analysis of studies using the STRATIFY tool for prediction of falls in hospital patients: How well does it work? Age and Ageing, 37(6), 621-627. doi: 10.1093/ageing/afn203

Ordem dos Enfermeiros. (2010). Programa padrões de qualidade dos cuidados de enfermagem. Recuperado de http://www.ordemenfermeiros.pt/projectos/Paginas/ PadroesdeQualidade.aspx
Papaioannou, A., Parkinson, W., CooK, R., Ferko, N., Coker, E., \& Adachi, J. D. (2004). Prediction of falls using a risk assessment tool in the acute care setting. BMC Medicine, 2(1), 1-7. doi:10.1186/1741-7015-2-1

Perell, K. L., Nelson, A., Goldman, R. L., Prieto-Lewis, N., \& Rubenstein, L. Z. (2001). Fall risk assessment measures: An analytic review. Journal of Gerontology, 56(12), 761-766. doi:10.1093/gerona/56.12.M761

Pina, S. M., Saraiva, D., Vaz, I., Ramalhinho, J., Ferreira, L., \& Batista, P. (2010). Quedas em meio hospitalar. Revista da Ordem dos Enfermeiros, 36, 27-29.

Schmid, N. A. (1990). Reducing patints falls: A research-based comprehensive fall prevention program. Military Medicine, 155(2), 202-207.

Schwendimann, R., De Geest, S., \& Milisen, K. (2006). Evaluation of the Morse Fall Scale in hospitalized patients. Age and Ageing, 35(3), 311-313. doi:10.1093/ageing/afj066

Soares, M. E., \& Almeida, M. R. (2008). Acidentes com macas e camas em estabelecimentos hospitalares, envolvendo a queda de doentes (Relatório n. ${ }^{\circ}$ 319/08). Lisboa, Portugal: Inspecção Geral das Actividades em Saúde.

Wild, D., Grove, A., Martin, M., Eremenco, S., McElroy, S., VerjeeLorenz, A., \& Erikson, P. (2005). Principles of good practice for the translation and cultural adaptation process for patient-reported outcomes (PRO) measures: Report of the ISPOR task force for translation and cultural adaptation. Value In Health, 8(2) 94-104. 
